\title{
Homocysteine activates autophagy by inhibition of CFTR expression via interaction between DNA methylation and H3K27me3 in mouse liver
}

Anning Yang ${ }^{1,2}$, Yun Jiao ${ }^{3}$, Songhao Yang ${ }^{1,2}$, Mei Deng ${ }^{1,2}$, Xiaoling Yang ${ }^{1,2}$, Caiyan Mao ${ }^{1,2}$, Yue Sun ${ }^{1,2}$, Ning Ding ${ }^{1,2}$, Nan Li ${ }^{4}$, Minghao Zhang ${ }^{1,2}$, Shaoju Jin ${ }^{4}$, Huiping Zhang ${ }^{2,3}$ and Yideng Jiang ${ }^{1,2}$

\begin{abstract}
Elevated homocysteine (Hcy) levels have been reported to be involved in liver injury, and autophagy plays an important role in normal hepatic physiology and pathophysiology, but the mechanism underlying Hcy regulated autophagy is currently unknown. In this study, $\mathrm{CBS}^{+/-}$mice were fed with regular diet for 12 weeks to establish a hyperhomocysteinemia (HHcy) model and HL-7702 cells were treated with Hcy, we found that Hcy increases autophagy and aggravates liver injury by downregulation of cystic fibrosis transmembrane conductance regulator (CFTR) expression in vivo and in vitro. Overexpression of CFTR inhibited the formation of autophagosomes and the expression of autophagy-related proteins BECN1, LC3-II/I and Atg12, while the expression of p62 increased in Hcytreated hepatocytes and $\mathrm{CBS}^{+/-}$mice injected with lentivirus expressing CFTR. Further study showed that CFTR expression is regulated by the interaction of DNA methyltransferase 1 (DNMT1) and enhancer of zeste homolog 2 (EZH2), which, respectively, regulate DNA methylation and histone H3 lysine 27 trimethylation (H3K27me3). In conclusion, our study showed that Hcy activates autophagy by inhibition of CFTR expression via interaction between H3K27me3 and DNA methylation in the mouse liver. These findings provide new insight into the mechanism of Hcyinduced autophagy in liver injury.
\end{abstract}

\section{Introduction}

Homocysteine (Hcy) is a non-essential sulfhydryl-containing amino acid derived from methionine metabolism which takes place mainly in liver ${ }^{1}$. Dysregulation of methionine metabolism results in accumulation of Hcy in plasma, subsequently leading to liver injury ${ }^{2,3}$. Autophagy has emerged as a critical intracellular degradative pathway

\footnotetext{
Correspondence: Huiping Zhang (zhp19760820@163.com) or Yideng Jiang (jiangyd@nxmu.edu.cn)

'Department of Physiology and Pathophysiology, School of Basic Medical Sciences, Ningxia Medical University, Yinchuan 750004, China

${ }^{2}$ Ningxia Key Laboratory of Vascular Injury and Repair Research, Yinchuan, China

Full list of author information is available at the end of the article

Anning Yang and Yun Jiao contributed equally to this work.

Edited by $Y$. Wang.
}

that maintains cell function and survival through the degradation of cellular components such as organelles and proteins ${ }^{4}$. Recent reports suggested that autophagy plays a role in liver homeostasis and energy conservation $^{5,6}$; however, the underlying mechanism of Hcy regulated autophagy remain unclear.

The cystic fibrosis transmembrane conductance regulator $(C F T R)$ is a cAMP-regulated chloride and bicarbonate channel that contributes to ion balance and fluid transport in a number of epithelial cell types ${ }^{7,8}$. There were evidences that CFTR regulates bile secretion and other functions at the apical membrane of biliary epithelial cells ${ }^{9}$. Loss of functional CFTR expression is thought to disturb the balance between fluid secretion 
and absorption into the epithelial layer, leading to cystic fibrosis liver disease ${ }^{9,10}$. Accordingly, a depressed autophagy has previously been reported in prostate cancer cells due to knockdown of $C F T R^{11,12}$. Notably, liver is a major metabolic organ of Hcy, but there is no evidence to show the correlation between CFTR and hepatic autophagy induced by Hcy.

Epigenetic modifications such as DNA methylation and histone H3 lysine 27 methylation (H3K27me) can silence gene expression ${ }^{13,14}$. Hcy could act as a methyl donor during methylation of DNA and proteins ${ }^{15}$, growing evidence also suggests that Hcy may be involved in the interference of DNA methylation leading to the change of gene expression ${ }^{16}$. Our previous studies have found that Hcy could induce the hypomethylation of the FABP4 and upregulate its expression ${ }^{17}$. Furthermore, another important regulator of chromatin state is histone methylation, and which is catalyzed by a group of histone methyl transferases, such as EZH2, lysine 27 on histone H3 $(\mathrm{H} 3 \mathrm{~K} 27 \mathrm{me} 3)^{18}$. Some reports have shown that DZNep (an inhibitor of $S$-adenosyl-L-homocysteine hydrolase)induced cell death results from the reduction of EZH2 expression due to its degradation by the proteasome, and in turn, from the reduction of the H3K27me3 expres$\operatorname{sion}^{19,20}$. Recently, the cross-talk between DNA methylation and H3K27me3 histone mark has been reported in a number of organisms and both marks are known to be required for proper developmental progression ${ }^{21}$. And the interaction between DNA methylation and H3K27me3 on gene regulation came into our sight as a potential goal worthy of further investigation.

In this study, we found that autophagy plays an important role in hepatic injury induced by Hcy. We also revealed that CFTR is an important regulator of autophagy, the expression of CTFR is regulated by interaction between H3K27me3 and DNA methylation, presenting evidence of new biomarkers for hepatic injury.

\section{Results}

Hcy induces autophagy in mouse liver and hepatocytes

In order to know whether Hcy can induce hepatic autophagy, $\mathrm{CBS}^{+/-}$mice were fed with a regular diet to establish HHcy animal model. Plasma Hcy levels in $\mathrm{CBS}^{+/-}$mice were 3.46 times higher than that in $\mathrm{CBS}^{+/+}$ mice (Fig. 1a), indicating that hyperhomocysteinemia (HHcy) animal model was induced successfully. Next, we investigated the effects of Hcy on liver injury and found that the levels of serum indicators of liver function such as AST and ALT in $\mathrm{CBS}^{+/-}$mice are much higher than that in $\mathrm{CBS}^{+/+}$mice (Fig. 1a). Furthermore, the morphological changes of livers were assessed by H\&E staining and Oil Red O. As shown in Fig. 1c, intact lobe structure with tidy hepatic cords and no degeneration, necrosis, or inflammatory cell infiltration were observed in $\mathrm{CBS}^{+/+}$mice.
Meanwhile, a considerable mass of fat vacuoles or deposition in hepatic tissue accompanied with mononuclear cells infiltration alongside the portal area and central vein was shown in liver of $\mathrm{CBS}^{+/-}$mice, as well as ballooning and hydropic degeneration of hepatocytes. Oil Red O staining revealed much greater hepatic steatosis (fat deposition) in liver of $\mathrm{CBS}^{+/-}$mice. Detection of $\mathrm{CBS}^{+/-}$mice liver by transmission electron microscope (TEM) showed abundant autophagic vacuoles sequestrating cytoplasm and organelles, such as mitochondria and endoplasmic reticulum. Double-membranes, autophagosomes filled with degraded organelles and autolysosomes were frequently observed (Fig. 1d). Further detection of autophagy-related proteins expression showed that HHcy enhances the ratio of LC3-II/I and the expression of BECN1 and Atg12, and inhibits the expression of p62 in the $\mathrm{CBS}^{+/}$mice $(P<0.01$, Fig. 1e, f). All these results suggest that HHcy induces liver autophagy in $\mathrm{CBS}^{+/-}$mice.

To evaluate the most effective concentration of Hcy, hepatocytes were treated with Hcy at different concentrations $(50-500 \mu \mathrm{mol} / \mathrm{L})$ for $24 \mathrm{~h}$. Cell death was significantly increased in Hcy $(100-500 \mu \mathrm{mol} / \mathrm{L})$-treated cells after $24 \mathrm{~h}$ compared to untreated cells (Fig. 1g), and the activities of AST and ALT were upregulated in hepatocytes after treatment with Hcy in a concentration dependent manner (Fig. 1h). Notably, we found that 100 $\mu \mathrm{mol} / \mathrm{L}$ of Hcy inhibits the cell viability to a minimal level, thus this dose was used for further experiments, as described below. Additionally, mRFP-GFP-LC3 adenoviral vectors were used to evaluate the autophagic level in cells treated with Hcy. Ad-mRFP-GFP-LC3, a specific marker for autophagosomes and autolysosomes, was transfected to hepatocytes. As expected, we found that cells treated with Hcy shows typically dense accumulation of mRFP-LC3 and GFP-LC3 puncta in the perinuclear region and cytoplasm. The numbers of mRFP-GFP-LC3 puncta dramatically increased in the cells upon Hcy treatment (Fig. 1i). Meanwhile, the ratio of LC3-II/I and the expression of BECN1 and Atg12 were upregulated and the expression of p62 was downregulated in hepatic cells treated with Hcy (Fig. 1j, k). The results suggested that Hcy induces autophagy of hepatic cells both in vivo and in vitro.

\section{Downregulation of CFTR plays a key role in Hcy induced hepatic autophagy}

For verifying whether CFTR is involved in liver autophagy, CFTR mRNA and protein expression were analyzed by qRT-PCR and western blot in liver of $\mathrm{CBS}^{+/-}$mice and hepatic cells treated with Hcy. We found that CFTR mRNA and protein expression decreased both in vivo and in vitro $(P<0.05$, Fig. $2 \mathrm{a}-\mathrm{d})$. To further explore the role of CFTR in autophagy, the hepatocytes were treated with an 

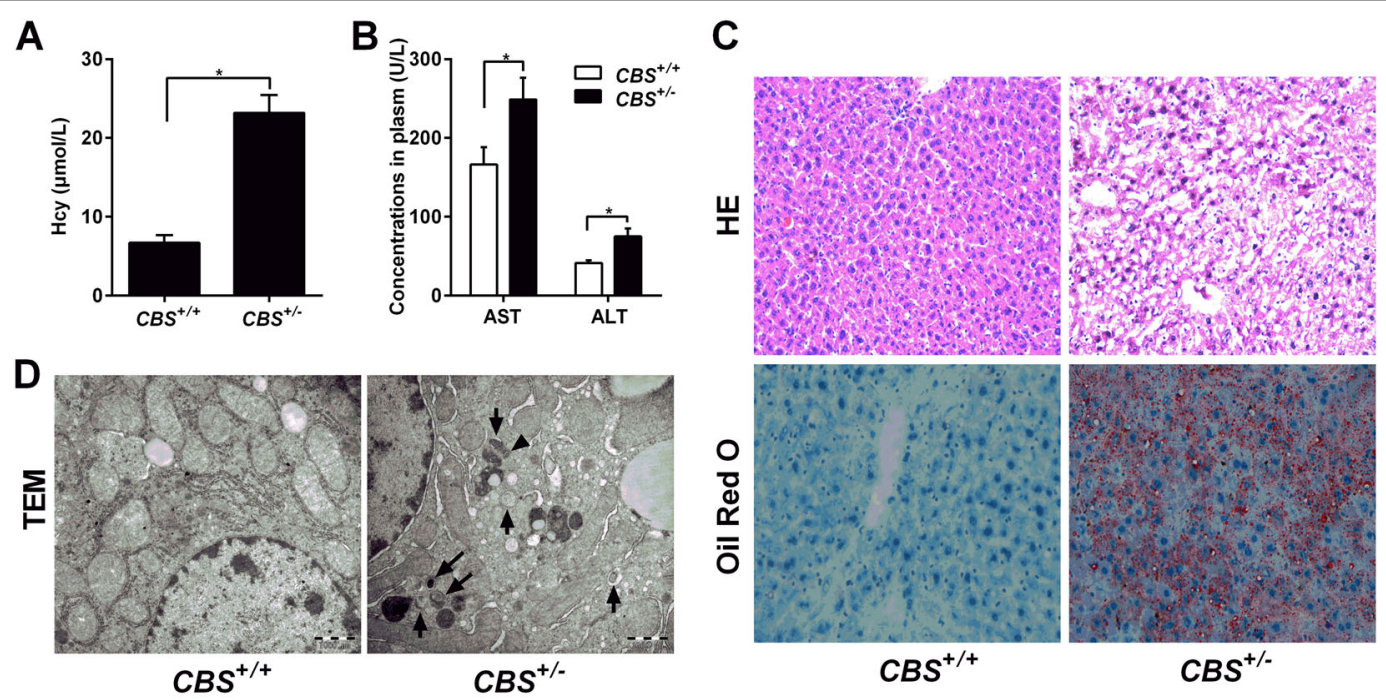

E

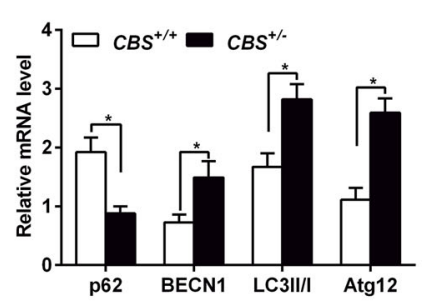

F

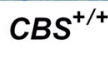

$\mathrm{CBS}^{+/-}$

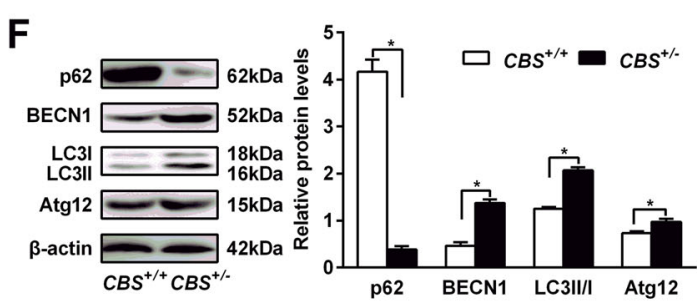

G
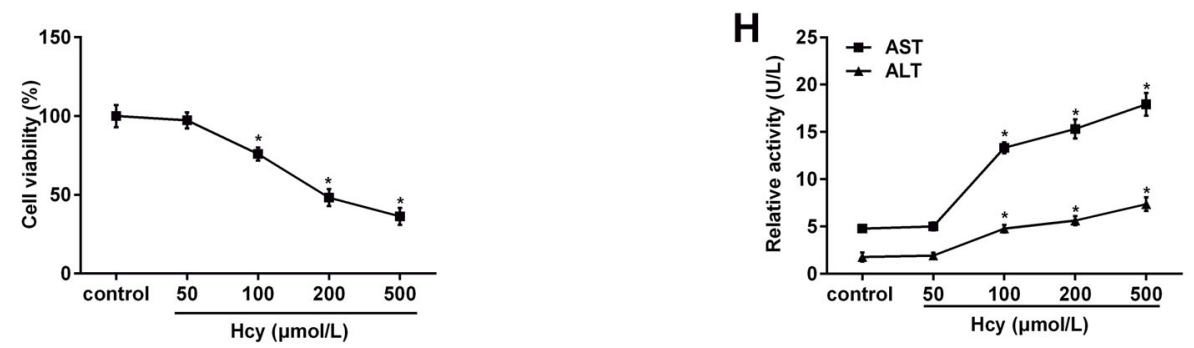

I
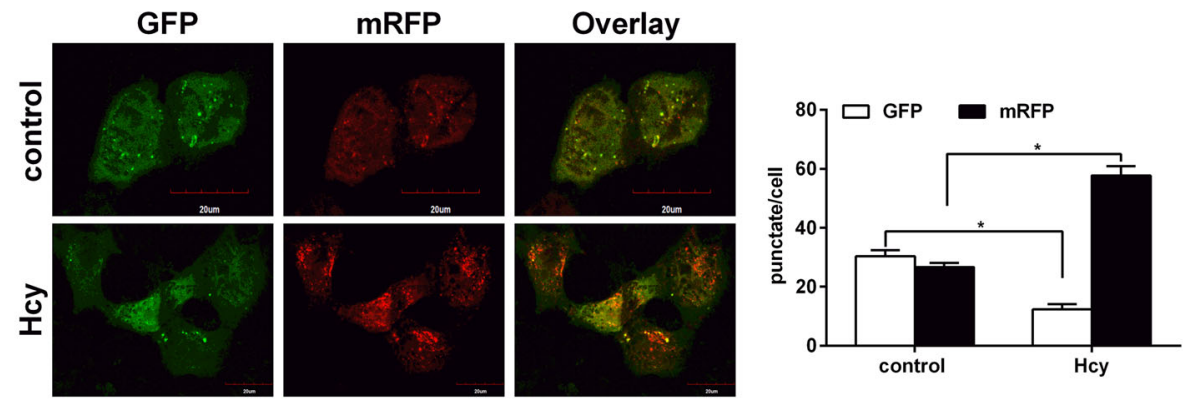

$\mathbf{J}$

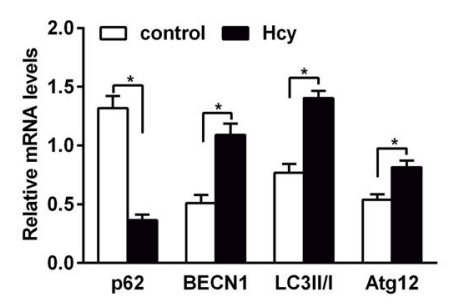

K

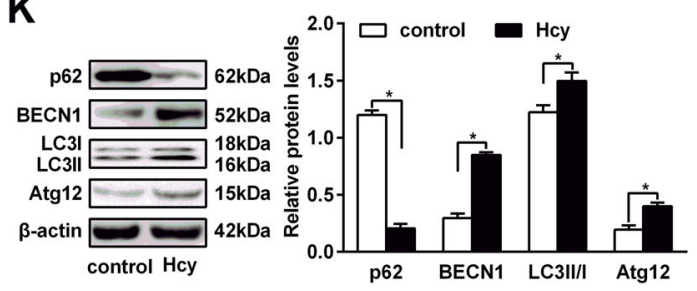

Fig. 1 (See legend on next page.) 
(see figure on previous page)

Fig. 1 Hcy induces hepatic autophagy in $\mathrm{CBS}^{+/-}$mice. Eight to 10 weeks old cystathionine b-synthase (CBS) heterozygous knockout mice (CBS ${ }^{+/-}$) were fed with regular mice chow and water ad libitum. Human hepatocytes HL-7702 were treated with L-Hcy (100 $\mu$ mol/L) for $24 \mathrm{~h}$. a The concentration of Hcy in plasma of mice was measured by automatic biochemical analyzer. $\mathbf{b}$ Contents of ALT and AST in serum of CBS ${ }^{+/-}$mice were analyzed using automatic biochemical analyzer. c Hematoxylin and eosin (H\&E) and Oil Red O staining of $\mathrm{CBS}^{+/-}$mice liver. d Transmission electron microscope (TEM) was used to analyse cell autophagy in liver tissues of $\mathrm{CBS}^{+/-}$mice. The arrows indicate the double-membrane vacuoles digesting organelles or cytosolic contents. e and $\mathbf{f}$ mRNA and protein expression of p62, BECN1, LC3 and Atg12 in the liver tissue of CBS ${ }^{+/-}$mice by qRT-PCR and western blot, respectively. $\mathbf{g}$ Hepatocytes were treated with different concentrations of Hcy $(50-500 \mu \mathrm{mol} / \mathrm{L})$ for $24 \mathrm{~h}$ before MTT assay. $\mathbf{h}$ The activities of AST and ALT in hepatocytes after treatment with Hcy were detected by ELISA. (I) Confocal fluorescent microscopy analysis of hepatocytes overexpressing mRFP-GFP-LC3, treated with $100 \mu \mathrm{mol} / \mathrm{L}$ Hcy for $24 \mathrm{~h}$. Quantification of mean red and green fluorescent puncta of at least 10 cells per condition is shown. The efficiency of transfection was also shown. $(\mathbf{J})$ and $(\mathbf{K})$ mRNA and protein expression of p62, BECN1, LC3 and Atg12 in hepatic cells treated with $100 \mu \mathrm{mol} / \mathrm{L}$ L-Hcy by qRT-PCR and western blot. Densitometry analysis of the proteins was performed for each sample (mean \pm s.d.). ${ }^{*} P<0.05$

potentiator, ivacaftor (VX-770) or antagonist (CFTR(inh)172), the results showed that the ratio of LC3-II/I and the expression levels of autophagy-related proteins BECN1 and Atg12 decreased and p62 increased in hepatocytes treated with VX-770. The effects of VX-770 are ameliorated $(P<0.05$, Fig. 2e, f) when hepatocytes were treated with Hcy. In contrast, the ratio of LC3-II/I and the expression levels of BECN1 and Atg12 increased and p62 decreased in hepatocytes treated with CFTR(inh)-172. The effects of CFTR(inh)-172 were enhanced when hepatocytes were treated with Hcy $(P<0.05$, Fig. $2 \mathrm{~g}, \mathrm{~h})$. Furthermore, to identify the effects, an adenoviral vector was constructed to overexpress CFTR in hepatocytes. CFTR protein expression was upregulated compared to the hepatocytes infected with adenoviral vector carrying a scrambled sequence. And on this basis, Hcy could downregulate the expression of CFTR in the hepatic cells $(P<0.05$, Fig. 2i). To test the effects of overexpression of CFTR in hepatocytes on the expression of autophagyrelated proteins p62, BECN1, LC3, and Atg12, we detected their expression after infection. The results are similar as the treatment with the agonist of CFTR, VX-770 $(P<$ 0.05 , Fig. 2j, k). This implies that Hcy treatment may repress CFTR transcription in the liver tissue of $\mathrm{CBS}^{+/-}$ mice and hepatocytes treated with Hcy.

\section{Augmentation of CFTR expression in the liver by in vivo lentivirus administrion reduces hepatic autophagy and liver injury in $\mathrm{CBS}^{+/-}$mice}

To know whether promoting CFTR expression could be an important intervention to protect liver against Hcyinduced autophagy, $C B S^{+/-}$mice were injected with lentivirus expressing CFTR (Lv-CFTR) (tilter $=2 \times 10^{7} \mathrm{TU} /$ $\mathrm{mL}$ ), lentivirus expressing GFP (Lv-GFP) (tilter $=2 \times 10^{7}$ $\mathrm{TU} / \mathrm{mL}$ ) or PBS by the tail vein, and after 30 days, mRNA and protein were isolated from livers. As Fig. 3a shows, both CFTR mRNA and protein expression were increased in the liver of Lv-CFTR-injected mice compared with control mice. Subsequently, we analyzed the level of aminopherase (AST and ALT). We found that aminopherase (AST and ALT) levels decrease in the sera of Lv-CFTR-injected mice as compared with mice injected with Lv-GFP (Fig. 3b). Meanwhile, we examined the histopathology of the livers by H\&E (Fig. 3c upper) and Oil Red O staining (Fig. 3c middle). Lv-GFP-injected mice had inflammatory cell infiltrates surrounding the portal and central veins, and extensive liver damage, hepatic steatosis. By contrast, livers from most Lv-CFTR-injected mice demonstrated significant attenuation of all of these pathological changes.

Further experiment was performed to investigate the relationship between CFTR and the hepatic autophagy, and TEM showed that autophagic vacuoles and autophagosomes reduces significantly in liver of Lv-CFTRinjected mice (Fig. $3 \mathrm{c}$ under). In addition, we analyzed the expression of autophagy related protein (p62, BECN1, LC3, and Atg12) in Lv-CFTR-injected mice models. Overexpression of CFTR significantly reduced the ratio of LC3-II/I and the expression of BECN1 and Atg12, increased the expression of p62 (Fig. 3d, e). All these results suggest that CFTR overexpression attenuated $\mathrm{HH} c y$ induces liver injury and autophagy in $\mathrm{CBS}^{+/-}$mice.

\section{DNMT1 positively regulates CFTR promoter methylation in Hcy-treated hepatocytes}

To explore whether Hcy regulates CFTR expression through CpG methylation, CpG island ( $-572 \mathrm{bp} /-262 \mathrm{bp})$ was predicted in the region of CFTR promoter using Methprimer software (Fig. 4a). The methylation status within CpG islands in CFTR promoter were detected by bisulfite sequencing PCR (BSP). As shown in Fig. 4b, c, CFTR DNA methylation in the liver of $\mathrm{CBS}^{+/-}$mice is higher than that in the liver of the $\mathrm{CBS}^{+/+}$mice. In hepatocytes, Hcy treatment enhances the DNA methylation of CFTR promoter as well, and the inhibition can be partly reversed by 5 -azacytidine treatment (Fig. $4 \mathrm{~d}$, e). Accordingly, these results were associated with a low expression of CFTR mRNA and protein (Fig. 4f).

To further know the role of DNMTs in promoter methylation of CFTR both in vivo and in vitro, we 


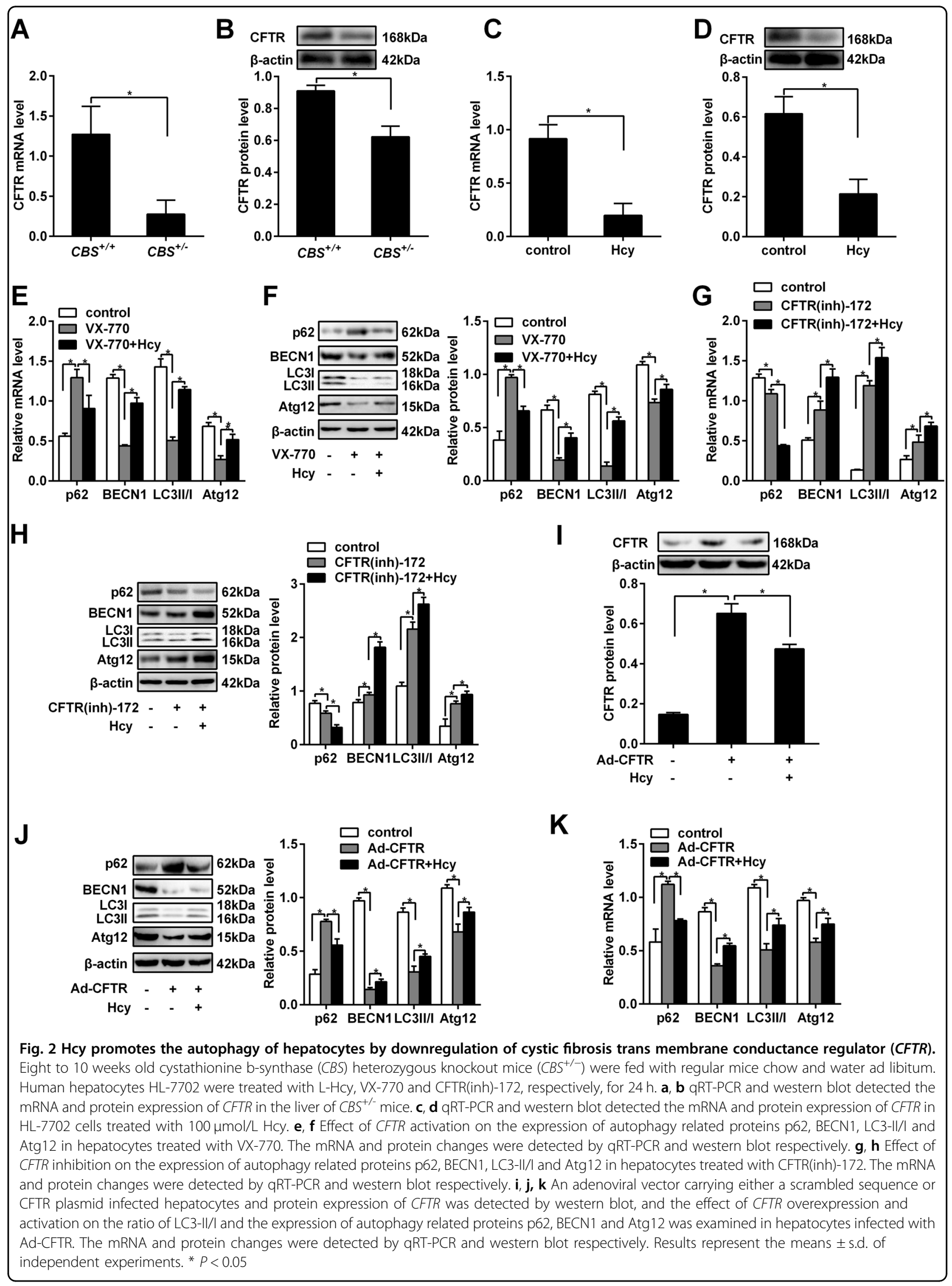



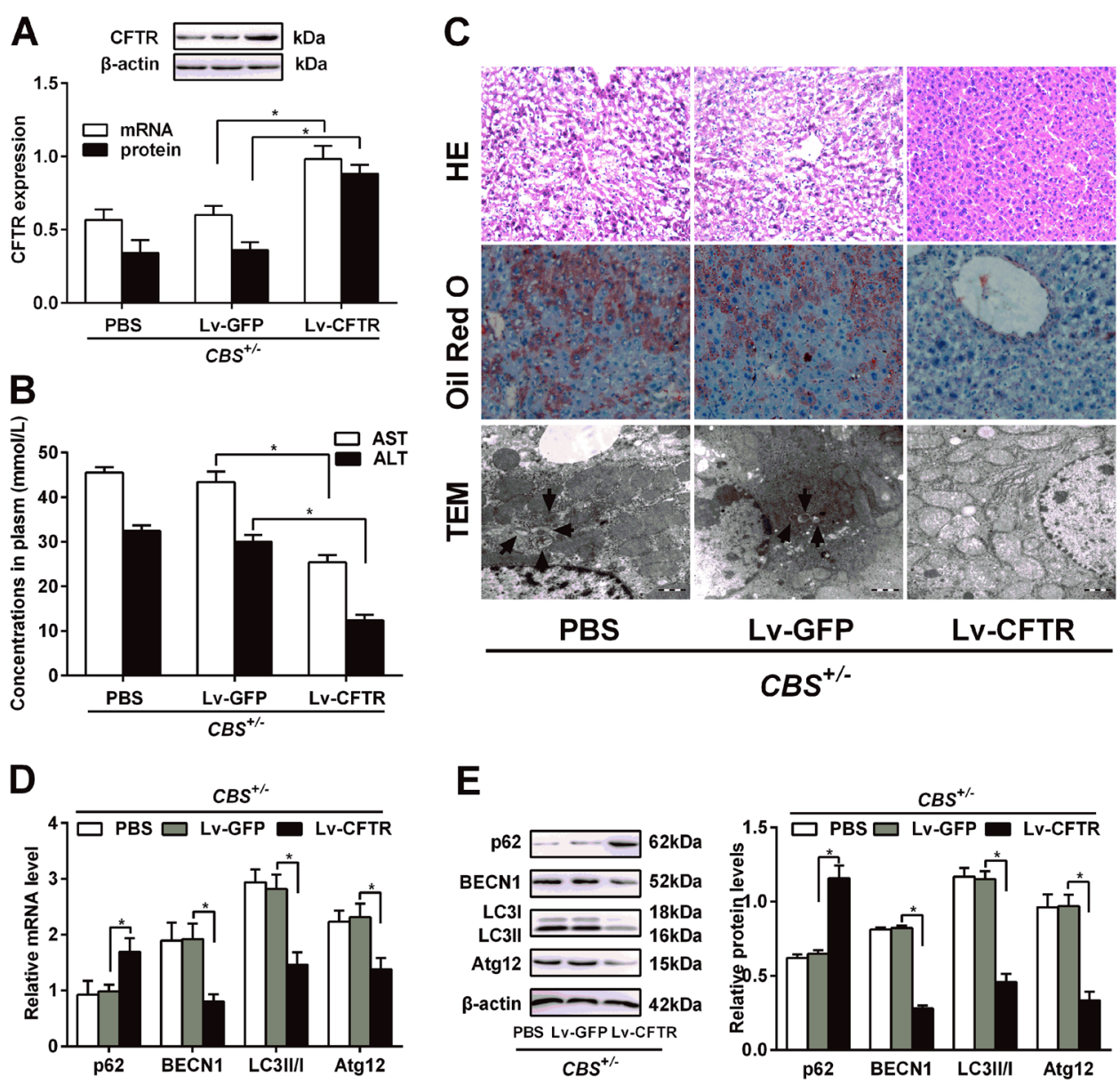

Fig. 3 CFTR alters the levels of liver injury biomarker and autophagy in $C B S^{+/-}$mice. $C B S^{+/-}$mice injected with lentivirus LV-CFTR or LV-GFP through the tail vein (see Materials and Methods). a CFTR mRNA and protein levels were determined by qRT-PCR and western blot respectively. Data are presented as mean \pm s.d. relative to Lv-GFP-injected mice. $\mathbf{b}$ Circulating levels of ALT and AST were analyzed. Results are means \pm s.d. ( $n=6$ ). ${ }^{*} P<$ 0.05. c Liver section from mice were subjected to H\&E (upper) and Oil Red O (middle) staining. Transmission electron microscope (TEM) (under) was used to analyse cell autophagy in liver of $\mathrm{CBS}^{+-}$mice. $\mathbf{d}$ and $\mathbf{e}$ mRNA and protein expression of p62, BECN1, LC3 and Atg12 were determined by qRT$\mathrm{PCR}$ and western blot respectively in the liver of $\mathrm{CBS}^{+/-}$mice after injection with lentivirus expressing CFTR

examined expression of DNMT1, 3a and 3b. The results indicated that DNMT1 mRNA expression is elevated in the liver of $\mathrm{CBS}^{+/-}$mice, which is consistent with protein quantification results $(P<0.01$, Fig. $4 \mathrm{~g}$, h). On the contrary, there is almost no difference in the expression of DNMT3a and DNMT3b in the liver between $\mathrm{CBS}^{+/-}$mice and $\mathrm{CBS}^{+/+}$mice. To further investigate whether DNMT1 regulates hypermethylation of CFTR promoter, we knocked down DNMT1 expression by adenovirus harboring DNMT1 small interfering RNA (si-DNMT1) in HL-7702 cells (Fig. 4i). Adenovirus si-DNMT1 can alleviate the upregulation of DNA methylation in the regions of CpG island of CFTR in Hcy-treated HL-7702 cells (Fig. $4 d, j$ ). Next, to verify whether DNMT1 could promote autophagy through downregulated CFTR expression in hepatocytes, we co-infected Ad-CFTR and si-DNMT1 into hepatocytes pre-treated by Hcy. The result showed that the ratio of LC3-II/I and the expression of BECN1, and Atg12 decreased and the expression of p62 increased in hepatocytes co-infected with Ad-CFTR and si-DNMT1 (Fig. 4k). Taken together, these data indicate that DNMT1 hypermethylates CFTR promoter enhances the level of autophagy in Hcy-treated hepatocytes.

\section{Hcy-induced elevation of EZH2 is responsible for CFTR promoter H3K27me3}

To study whether histone H3 lysine 27 (H3K27) methylation regulates CFTR expression, we detected H3K27 methylation on CFTR promoter by ChIP-PCR. The results demonstrated trimethylated H3K27 (H3K27me3) in the proximal promoter region of CFTR in the liver of $\mathrm{CBS}^{+/-}$mice, but not H3K27me1 and H3K27me2, is higher than that in the liver of $\mathrm{CBS}^{+/+}$ mice (Fig. 5a). Meanwhile, similar results were also 


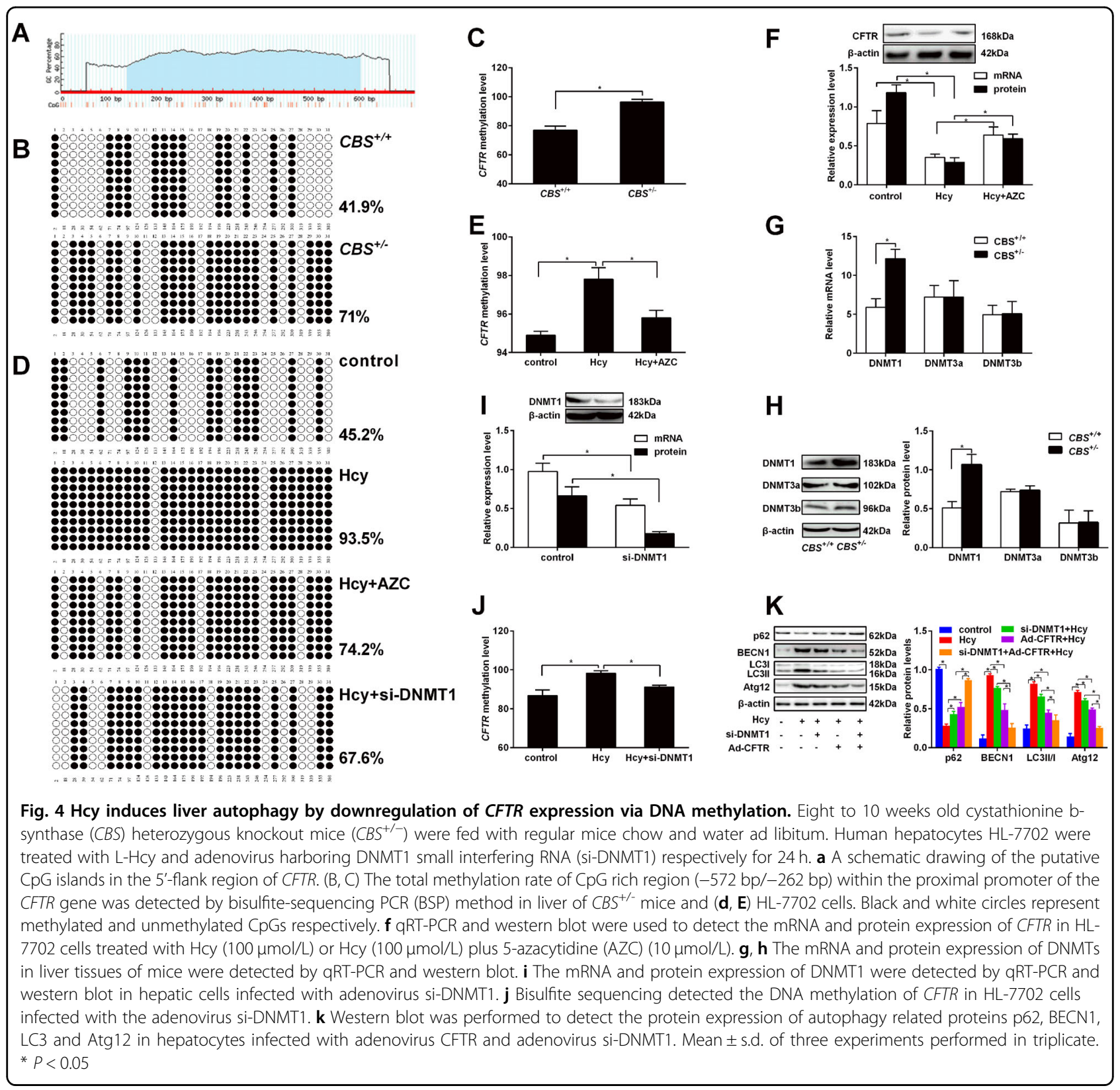

observed at the cell level, with increased level of H3K27me3 detected in the hepatocytes treated with Hcy (Fig. 5b). To check the inhibiting effect of EPZ005687 on H3K27me3, we detected the level of H3K27me3 in hepatocytes after treatment with different concentration EPZ005687. Western blot showed that downregulation of H3K27me3 level is positively correlated with the concentration of the EPZ005687 (Fig. 5c). Furthermore, Hcy treatment of HL-7702 cells inhibited CFTR expression as well, and the downregulation can be partly reversed by EPZ005687 treatment. (Fig. 5d, e), suggesting a possible role of H3K27me3 in the regulation of CFTR expression in Hcy treated liver.
We also tested impact of Hcy on the expression of EZH2 and H3K27me3 in CFTR promoter. The results showed that protein expression of EZH2 was enhanced in the liver of $\mathrm{CBS}^{+/-}$mice (Fig. 5f, g) and HL-7702 cells treated with Hcy (Fig. 5h, i). Knockdown of EZH2 by adenovirus harboring EZH2 small interfering RNA (siEZH2) in HL-7702 cells inhibited H3K27me3 in CFTR promoter (Fig. 5j) and these results were associated with a high expression of CFTR protein (Fig. 5k). The result suggests that Hcy downregulates the expression of CFTR through enhanced CFTR promoter H3K27me3 by EZH2.

Based on the results above, whether EZH2 could promote autophagy in hepatocytes was further studied. The 


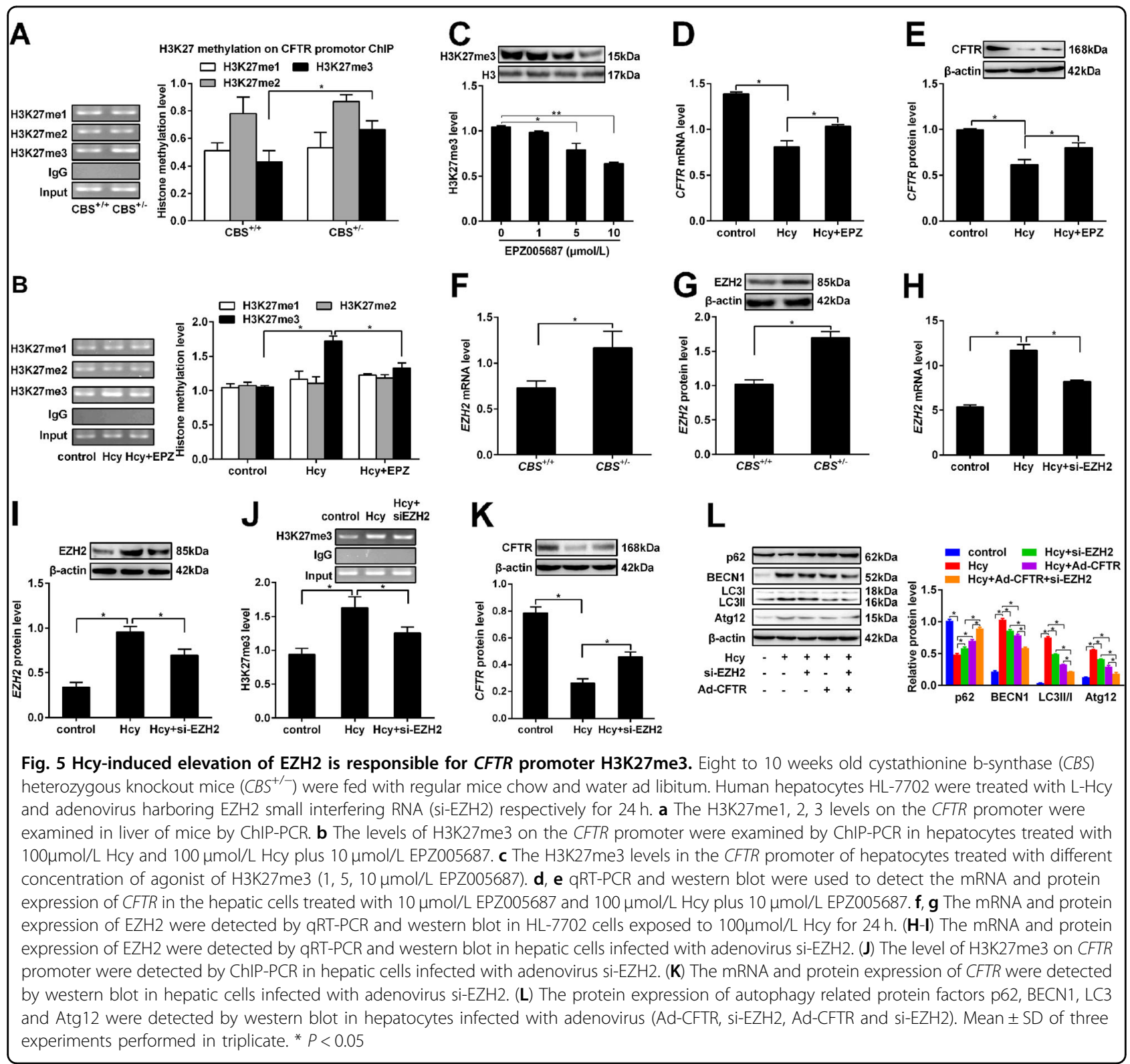

result showed that the ratio of LC3-II/I and the expression of autophagy-related proteins BECN1 and Atg12 decreased and the expression of p62 increased in hepatocytes co-infected with Ad-CFTR and si-EZH2 (Fig. 5l). Taken together, these data indicate that EZH2 hypermethylates CFTR promoter H3K27me3 enhances the level of autophagy in Hcy-treated hepatocytes.

CFTR promoter DNA methylation and histone methylation interact with each other in hepatocyte autophagy induced by Hcy

As an inhibitor of histone lysine methyltransferase that can inhibit histone methylation, EPZ005687 inhibited DNA methylation of CFTR promoter in addition to the inhibition of histone methylation in HL-7702 cells (Fig. 6a, b, d). Meanwhile, DNA methyltransferase inhibitor 5-azacytidine (AZC) can inhibit H3K27me3 of CFTR promoter in addition to the inhibition of DNA methylation of CTFR promoter (Fig. 6a, b, d), suggesting that DNA methylation and histone methylation of CTFR promoter could correlate with each other. To further confirm that, EZH2 and DNMT1 that mediate the histone methylation and DNA methylation in hepatocytes were knocked down respectively. The results showed that knockdown of either EZH2 or DNMT1 can repress both DNA methylation and histone methylation (Fig. 6c, e). To elucidate these effects on the regulation of autophagyrelated protein expression in hepatocytes, we perform 


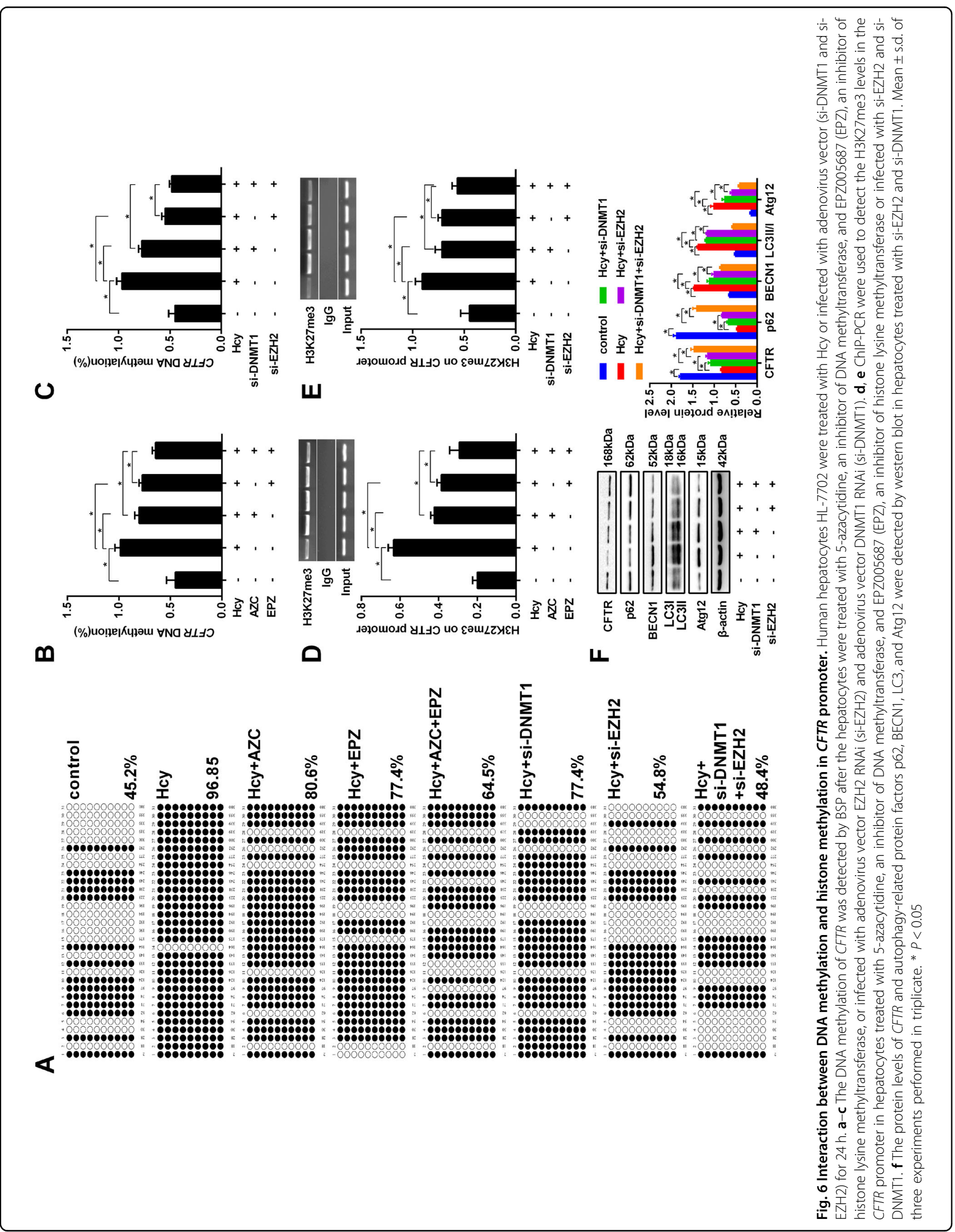


western blot and found that the ratio of LC3-II/I and the expression of autophagy-related proteins BECN1 and Atg12 decreased and the expression of $\mathrm{p} 62$ and CFTR increased in hepatocytes co-infected with si-EZH2 and siDNMT1 (Fig. 6f). All these data suggest that DNA methylation and histone methylation interaction may play a vital role in CFTR downregulation, which further mediates Hcy-induced hepatic autophagy.

\section{Discussion}

In the current study, we examined the biological effects of elevated Hcy and the involvement of CFTR in Hcyinduced autophagy. Our findings proved a cross-talk between DNA methylation and histone methylation in the regulation of CFTR expression, leading to hepatic autophagy induced by Hcy. The results provide fundamental insight into how autophagy homeostasis is maintained.

Dysfunction of liver alters methionine metabolism, resulting in elevated Hcy that is released into the plasma $^{22}$. In return, Hcy can influence the status of liver as well, and autophagy has been proposed to explain the pathogenic effects of Hcy in hepatic injury ${ }^{23}$. In this study, we found that autophagy occurrs in $\mathrm{CBS}^{+/-}$mice liver. Additionally, similar results were found in Hcy-treated hepatocytes. The recent discovery of Hcy-triggered autophagy contributes to brain neuronal cell injury following focal cerebral ischemia-reperfusion with the generation of autophagosomes and the upregulation of LC3B/ $\mathrm{BECN1}$ protein expression in neurons ${ }^{24}$. The possible explanation is that the oxidative damage-mediated autophagy may be a molecular mechanism underlying hepatocellular damage by elevated Hcy level, which further confirms our results that Hcy promotes autophagy to induce liver injury.

Recent study reported that CFTR orchestrates a proteostatic network that influences multiple cellular functions including autophagy by acting as a hub protein ${ }^{25,26}$. Our results demonstrated that CFTR expression decreases in $\mathrm{CBS}^{+/-}$mice, which is consistent with that CFTR expression in mice is downregulated during a much later stage of tunicamycin-induced autophagy. Then the expression of p62, BECN1, LC3 and Atg12 were measured in CFTR-overexpressed hepatocytes. Results of the present study revealed that, in the presence of Hcy, autophagy was enhanced when transcriptional activation of CFTR was reduced in hepatocytes. Overexpression of CFTR inhibited the formation of autophagosomes and the expression of autophagy-related proteins BECN1, LC3-II/ I, and Atg12, while the expression of p62 increased in Hcy-treated hepatocytes and $C B S^{+/-}$mice injected with lentivirus expressing CFTR. A previous study provided striking molecular evidence that etiologic CFTR mutations trigger a cascade of events culminating in the autophagy-related protein BECN1 depletion and impairment caused by autophagy ${ }^{27}$. Additionally, Tosco A et al. ${ }^{28}$ found that correct of dysfunctional CFTR may be the most intellectually satisfying way for therapeutic strategy in cystic fibrosis, by restore autophagy, all which support our observations that CFTR plays a crucial role in hepatocyte autophagy. One of the traditional metabolic pathways of Hcy is sulfur transfer way, and CFTR has the ability to form disulfide bond. It has been recently reported that Hcy may transfer sulfhydryl group to other substances (such as CFTR) to cause hepatic autophagy, which might be a novel mechanism for Hcy-induced liver injury. To our confusion, Hcy is a non-essential sulfhydryl-containing amino acid derived from methionine metabolism, it is dynamically regulated by re-methylation and trans-sulfuration pathway. It is not comprehensive to explain the pathogenic mechanism of Hcy only by means of trans-sulfuration pathway, which needs to be further explored.

Elevated levels of Hcy can alter global DNA methylation and induce promoter specific methylation in many genes implicated in human diseases including cancer, neurodegenerative diseases and cardiovascular disease ${ }^{29-31}$. Earlier reports ${ }^{32}$ have demonstrated that Hcy triggers inflammatory responses in macrophages through inhibiting CSE- $\mathrm{H}_{2} \mathrm{~S}$ signaling via DNA hypermethylation of CSE promoter. Our previous work also indicated that DNA hypermethylation suppresses p53 expression in cardiac tissue of $A p o E^{-/-}$mice with $\mathrm{HHcy}^{33}$. In present work, we found that DNA hypermethylation represses CFTR transcription in $\mathrm{CBS}^{+/-}$mice liver which has high levels of Hcy. Furthermore, CFTR DNA methylation level was increased in hepatocytes co-incubated with Hcy. We also found an increase of DNMT1 expression both in $\mathrm{CBS}^{+/-}$mice and hepatocytes. Since Hcy is a precursor of $S$-adenosyl methionine (SAM) which is a universal methyl donor, high level of Hcy can lead to high level of SAM. In the meantime, SAM is one of the substrates for DNMTs, high levels of SAM increase the activity of DNMTs, which is reflected in the global methylation experiment. Apart from DNA methylation, Hcy is associated with histone methylation, which alters the expression of remodeling genes $^{34}$. Our study showed an increase in H3K27me3 on CFTR promoter and the expression of EZH2 in $\mathrm{CBS}^{+/-}$ mice. Accordingly, treating hepatocytes with Hcy resulted in promoted H3K27me3, CFTR hypermethylation, and silencing of CFTR. To explicit the relationship between EZH2 and DNMT1, si-DNMT1 and si-EZH2 were cotransfected into hepatocytes pre-treated by Hcy, we found downregulated CFTR DNA methylation, decreased H3K27me3 and increased expression of CFTR. These results suggested that interaction between EZH2 and DNMT1 downregulates CFTR expression in hepatocytes treated with Hcy. There are a few mechanisms supporting our findings. First, methylation of DNA and histone use a 
common methyl donor (i.e., SAM). In addition, modifications of DNA and histone methylation are carefully to modulate gene expression programming in the organism through direct interactions between histone and DNMTs. Alterations in intracellular availability of SAM can result in changes in gene expression. Furthermore, many studies suggested that Hcy can also directly affect the epigenetic regulation of gene expression ${ }^{35}$.

In conclusion, our data demonstrated a crucial role of $C F T R$ in reducing hepatic autophagy in the presence of Hcy. We also revealed that CFTR may mediate the effects of metabolic perturbations of Hcy-induced autophagy. DNA methylation and histone methylation regulate CFTR expression leading to autophagy induced by Hcy is a novel mechanism of liver injury. However, the underlying mechanism of CFTR in Hcy-induced hepatic autophagy needs to be further explored.

\section{Materials and methods \\ Animals}

All animal experiments were approved and carried out in accordance with the Institutional Animal Care and Use Committee of the University of Ningxia Medical University. Eight to 10 weeks old cystathionine beta-synthase $(C B S)$ heterozygous knockout mice $\left(\mathrm{CBS}^{+-}\right)$were obtained from Jackson Laboratory (Bar Harbor, ME) and maintained in the animal facility center at University of Ningxia Medical University. The mice were fed with regular mice chow and water ad libitum. Mice genotypes were determined by a PCR of DNA obtained from tail biopsies with a specific set of primers ${ }^{36}$. For evaluation of the therapeutic potential of recombinant lentiviruses expressing human CFTR (Lv-CFTR), 19 mice were injected with Lv-CFTR and Lv-GFP into the tail vein respectively, the titers averaged $2 \times 10^{7} \mathrm{TU} / \mathrm{mL}$, and total volume was $80 \mathrm{~mL}$. Equal volume of PBS was used as a vehicle control. Thirty days after lentivirus injection, mice were sacrificed and livers were flash-frozen in liquid $\mathrm{N}_{2}$ and processed to obtain total protein.

\section{Liver tissue preparation and morphologic observation}

Thirty days after treatment, all mice were fasted but supplied with water for $24 \mathrm{~h}$. Then, mice peritoneally injected with $3 \%$ pentobarbital sodium according to injection dose to mouse weight of $2 \mathrm{~mL} / \mathrm{kg}$. Abdominal cavity of anesthetic mouse was incised, and then inferior vena cava blood was collected using $10 \mathrm{~mL}$ syringe. After standing for $3 \mathrm{~h}$ at $4{ }^{\circ} \mathrm{C}$, blood samples were centrifuged at $5000 \times g$ for $15 \mathrm{~min}$. Supernatant was saved at $-80^{\circ} \mathrm{C}$ for future use. Liver was incised and two segments $(1.0 \mathrm{~cm} \times$ $1.0 \mathrm{~cm} \times 0.3 \mathrm{~cm}$ ) were cut from right lobe of the liver. Two liver segments were fixed in $10 \%$ neutral formalin and embedded in optimum cutting temperature compound (OCT). The remained liver samples were saved at $-80^{\circ} \mathrm{C}$ for future use. To observe morphologic changes in liver tissues, the HE staining and red $\mathrm{O}$ staining of liver segments was performed according to previous description $^{37,38}$.

\section{Plasmatic parameters estimation}

The plasmatic alanine aminotransferase (ALT) and aspartate transaminase (AST) activities were measured using the Cobas e411 analyzer (Roche Diagnostics, Mannheim, Germany), according to the controls and calibration of the Cobas e411 analyzer (Roche Diagnostics, Mannheim, Germany).

\section{Transmission electron microscopy (TEM)}

The livers were fixed in $0.1 \mathrm{M}$ cacodylate buffer containing $2.5 \%$ glutaraldehyde at $4{ }^{\circ} \mathrm{C}$ for at least $24 \mathrm{~h}$. The livers were then dissected into quadrants, osmicated, washed, block contrasted with $2 \%$ aqueous uranyl acetate, dehydrated through a graded series of ethanol, and embedded in Epon 812 (Plano GmbH, Marburg, Germany). Ultrathin sections (about $50 \mathrm{~nm}$ ) were mounted on pioloform-coated slot copper grids and contrasted with uranyl acetate $(5 \mathrm{~min})$ and lead citrate $(3 \mathrm{~min})$. The specimens were examined using a Zeiss EM 902 transmission electron microscope (Zeiss, Oberkochen, Germany) at $80 \mathrm{kV}$. Photographs were taken by a CCD camera (Proscan, Lagerlechfeld, Germany).

\section{Cell culture and virus transfection}

Human hepatocytes (HL-7702) were obtained from the Japanese Collection of Research Bioresources and cultured in RPMI-1640 medium (Thermo, USA) containing $10 \%$ fetal bovine serum (FBS) (Hyclone, USA) and 1\% penicillin (Thermo, Waltham, MA, USA) humidified with $5 \% \mathrm{CO}_{2}$ air at $37^{\circ} \mathrm{C}$. The cells were transfected with AdCFTR when they were $80 \%$ confluent. The control cells were transfected with Ad-GFP. After $48 \mathrm{~h}$, the transfection efficiency was detected by fluorescence microscope, and then the cells were treated with L-Hcy $(100 \mu \mathrm{mol} / \mathrm{L})$ (Sigma, USA) for another $24 \mathrm{~h}$ for further experiments.

\section{Quantitative real-time PCR}

Quantitative real-time PCR (qRT-PCR) for CFTR, DNMT1 and EZH2 genes was performed using an FTC3000 qRT-PCR detection system as described previously $^{17,39}$. qRT-PCR kits were from Takara Biotechnology Co., Ltd. (Dalian, China). The first-strand cDNA reaction $(0.5 \mu \mathrm{L})$ was subjected to qRT-PCR amplification using gene-specific primers (Table 1). All primers were synthesized by Sangon Biotech Co., Ltd. (Shanghai, China). qRT-PCR reaction was performed with an initial denaturation at $95^{\circ} \mathrm{C}$ for $10 \mathrm{~min}$ followed by 40 cycles of denaturation at $95^{\circ} \mathrm{C}$ for $20 \mathrm{~s}$, annealing at $58^{\circ} \mathrm{C}$ for $30 \mathrm{~s}$, and extension at $72{ }^{\circ} \mathrm{C}$ for $30 \mathrm{~s}$, and, for a final 


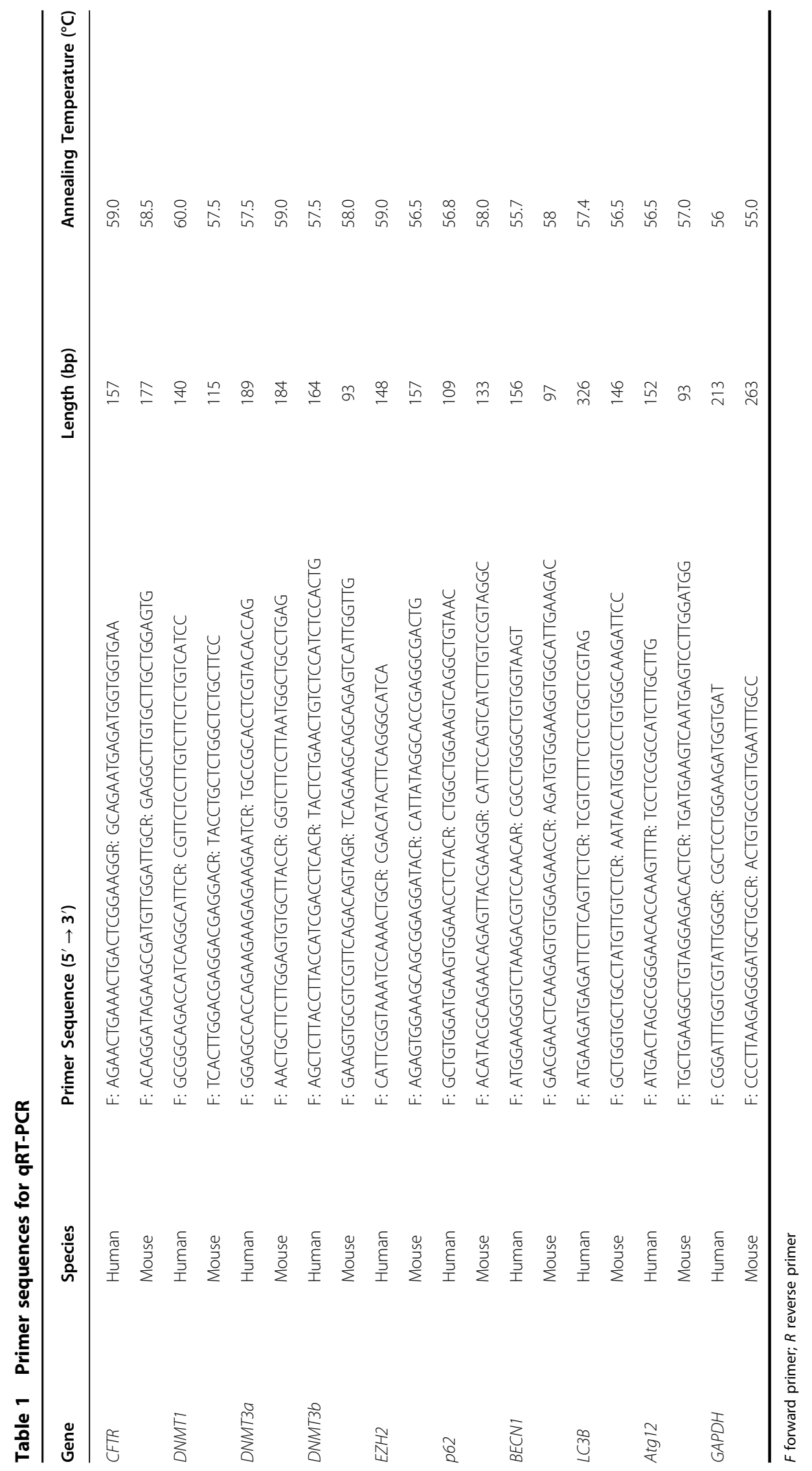


step, a melting curve of $94{ }^{\circ} \mathrm{C}$ for $90 \mathrm{~s}, 60^{\circ} \mathrm{C}$ for $180 \mathrm{~s}$, and $94{ }^{\circ} \mathrm{C}$ for $10 \mathrm{~s}$. Glyceraldehyde phosphate dehydrogenase (GAPDH) was used as the invariant control; mRNA expression was determined by $2^{-\Delta \Delta \mathrm{CT}}$ method. Results were obtained from triplicate experiments.

\section{Western blot}

Western blot analysis was performed as previously described $^{17,39}$. Murine liver tissues and HL-7702 cells were lysed in a lysis buffer (KeyGEN, China) containing the protease inhibitor phenylmethanesulfonyl fluoride (PMSF, KeyGEN, China) at $4{ }^{\circ} \mathrm{C}$ for $30 \mathrm{~min}$ followed by centrifugation to remove cell debris. Protein concentration was measured using BCA protein assay kit (Beyotime Institute of Biotechnology). The protein was boiled and subjected to western blot with antibodies against CFTR, DNMT1, DNMT3a, DNMT3b, EZH2, p62, BECN1, microtubuleassociated protein 1 light chain 3 (LC3), Atg12 and $\beta$-actin (all from Abcam Inc., Cambridge, MA, USA) respectively. Optical densities of the bands were analysed with Bio-Rad image analysis (Bio-Rad, Hercules, CA, USA).

\section{Cell viability assay}

Cell viability was determined by MTT assay as originally described by Mosmann et al. ${ }^{40}$. In brief, hepatic cells were plated on a 96 well plate at a density of $10^{5}$ cells/well and incubated with different concentrations of Hcy $(0-500$ $\mu \mathrm{M})$ in serum-free RPMI- 1640 at $37^{\circ} \mathrm{C}$ for $24 \mathrm{~h}$. Then MTT was added to each well followed by incubation for 4 $\mathrm{h}$ at $37^{\circ} \mathrm{C}$. The medium was removed and wells were rinsed twice with PBS. After that, $100 \mu \mathrm{l}$ of DMSO was added to each well to dissolve the cell membrane and bring formazan into the solution. The amount of formazan was then measured by collecting absorbance values at $570 \mathrm{~nm}$ by spectramax3000 spectrophotometer (Molecular Devices, Sunnyvale, CA).

\section{Autophagy detection using mRFP-GFP adenoviral vector}

mRFP-GFP-LC3 adenoviral vectors were purchased from HanBio Technology Co. Ltd. (HanBio, shanghai, China). Hepatocytes were seeded in six-well plates andthey were incubated in growth medium with the adenoviruses at a MOI of 100 for $2 \mathrm{~h}$ at $37^{\circ} \mathrm{C}$, and were then grew in medium with addition of $100 \mu \mathrm{mol} / \mathrm{L}$ Hcy for another $24 \mathrm{~h}$ at $37^{\circ} \mathrm{C}$. Autophagy was observed under a confocal fluorescence microscope (Olympus BX 51, Tokyo, Japan). Autophagic flux was determined by evaluating the number of GFP and mRFP puncta (puncta/cell were counted).

\section{Chromatin immunoprecipitation (ChIP) assays}

Formaldehyde (1\%) was added to the samples to crosslink protein-DNA complexes. ChIP trials were conducted using a ChIP assay kit (Merck Millipore, DA, Germany).
After cross-linking, the DNA was fragmented by sonication (UCD-200; Diagenode, Liège, Belgium), which consisted of 25 cycles of $30 \mathrm{~s}$ each with an interval of $30 \mathrm{~s}$ to cool down. Then, protein-DNA complexes were precipitated with an anti-H3K27me1, H3K27me2 and H3K27me3 (Abcam, MA, USA) antibody. DNA was extracted using a DNA purification kit (Merck Millipore). The experiment contained both a positive control group (precipitated with an anti-RNA polymerase II antibody) and a negative control group (precipitated with normal mouse IgG). The amount of extracted DNA was determined by qRT-PCR. The primers were designed as follows: the forward primer is: 5'CGACGGACGATCTATGATGC-3' and the reverse primer is: 5'-AGTCGCAGCTTCTCGTCAG-3'.

\section{Bisulfite sequencing}

DNA was extracted from the cells using a DNA extraction kit (AP-MN-MS-GDNA-250; Axygen, CA, USA) and then treated with bisulphate (ZYMO RESEARCH). The PCR products were gel extracted (APGX-250; Axygen) and ligated into a plasmid vector using the pMD18-T Vector (D101B; Takara). Plasmid transformed DH5a bacteria were cultured overnight, and the plasmid DNA was isolated (Axygen). At least 10 separate clones were chosen for sequence analysis.

\section{RNA interference}

Oligonucleotide sequences for RNA interference (RNAi) controls (nonsilencing), DNMT1 and EZH2 were as following: nonsilencing small interfering RNA (siRNA), UUCUCCGAACGUGUCACGU; DNMT1 siRNA, CACUGGUUCUGCGCUGGGA; EZH2 siRNA, AGCUCUUUGACGUGCAAUA. All RNAi oligonucleotides were purchased from Shanghai GeneChem Company (Shanghai) and transfected into HL-7702 cells using the Lipofectamine 2000 transfection kit (Invitrogen, Carlsbad, CA) according to the manufacturer's instructions.

\section{Statistical analysis}

Each experiment was performed 3 times. Single parameters between 2 groups was compared by the paired Student's $t$-test. One-way ANOVA was used to compare multiple groups, followed by Newman-Keuls test. $P$ values $<0.05$ are considered as statistically significant.

\section{Acknowledgements}

This work was supported by the National Natural Science Foundation of China (81360073, 81460121, 81560084, 81570452, 81660047, 81670416, 81660088), the Natural Science Foundation of Ningxia Province (NZ16149) and the Ningxia College Scientific Research Project (NGY2015071).

\section{Author' contributions:}

A.Y., Y.J., H.Z. and Y.J. conceived the experiment(S), A.Y.,Y.S., S.Y., C.M., N.L., N.D and M.D., conducted the experiment(S), A.Y., X.Y., M.Z., and S.J. analysed the results. All authors reviewed the manuscript. 


\section{Author details}

'Department of Physiology and Pathophysiology, School of Basic Medical Sciences, Ningxia Medical University, Yinchuan 750004, China. ${ }^{2}$ Ningxia Key Laboratory of Vascular Injury and Repair Research, Yinchuan, China. ${ }^{3}$ Ningxia Medical University General Hospital, Yinchuan 750004, China. ${ }^{4}$ Pharmacy college, Ningxia Medical University, Yinchuan 750004, China

\section{Conflict of interest}

The authors declare that they have no conflict of interest.

\section{Publisher's note}

Springer Nature remains neutral with regard to jurisdictional claims in published maps and institutional affiliations.

Received: 15 August 2017 Revised: 11 November 2017 Accepted: 13 December 2017

Published online: 07 February 2018

\section{References}

1. Wan, L., Sun, Y., Zhang, F. \& Ren, Y. Low-dose homocystine enhances proliferation and migration of Bv2 microglia cells. Cell. Mol. Neurobiol. 36, 1279-1289 (2016).

2. Zhou Z., Vailati-Riboni M., Luchini D. N. \& Loor J. J. Methionine and choline supply during the periparturient period alter plasma amino acid and onecarbon metabolism profiles to various extents: potential role in hepatic metabolism and antioxidant status. Nutrients 9, 10 (2017).

3. Jacobs, R. L. et al. Cystathionine beta-synthase deficiency alters hepatic phospholipid and choline metabolism: Post-translational repression of phosphatidylethanolamine $\mathrm{N}$-methyltransferase is a consequence rather than a cause of liver injury in homocystinuria. Mol. Genet. Metab. 120, 325-336 (2017).

4. Spirli, C. et al. Posttranslational regulation of polycystin-2 protein expression as a novel mechanism of cholangiocyte reaction and repair from biliary damage. Hepatology 62, 1828-1839 (2015).

5. Settembre, $C$. et al. TFEB controls cellular lipid metabolism through a starvation-induced autoregulatory loop. Nat. Cell Biol. 15, 647-658 (2013).

6. Sydor, S. \& Bechmann, L. P. Hepatic stellate cell autophagy in acute liver injury: A novel role for nitric oxide? Liver Int. 37, 1602-1604 (2017).

7. Gao, X.\& \&wang, T. C. Spatial positioning of CFTR's pore-lining residues affirms an asymmetrical contribution of transmembrane segments to the anion permeation pathway. J. Gen. Physiol. 147, 407-422 (2016).

8. Riordan, J. R. CFIR function and prospects for therapy. Annu. Rev. Biochem. 77 $701-726$ (2008)

9. Fiorotto, R. et al. The cystic fibrosis transmembrane conductance regulator controls biliary epithelial inflammation and permeability by regulating Src tyrosine kinase activity. Hepatology 64, 2118-2134 (2016).

10. Grasemann, H. CFTR Modulator Therapy for Cystic Fibrosis. N. Engl. J. Med. 377 2085-2088 (2017).

11. Zhu, Q., Li, H., Liu, Y. \& Jiang, L. Knockdown of CFTR enhances sensitivity of prostate cancer cells to cisplatin via inhibition of autophagy. Neoplasma 64, 709-717 (2017)

12. Xie, C. et al. CFTR suppresses tumor progression through miR-193b targeting urokinase plasminogen activator (UPA) in prostate cancer. Oncogene $\mathbf{3 2}$ 2282-2291 (2013). 2291 e2281-2287.

13. Kim, J. et al. Increased expression of the histone $\mathrm{H} 3$ lysine 4 methyltransferase MLL4 and the histone H3 lysine 27 demethylase UTX prolonging the overall survival of patients with glioblastoma and a methylated MGMT promoter. J. Neurosurg. 126, 1461-1471 (2017)

14. Choi, J. et al. DNA binding by PHF1 prolongs PRC2 residence time on chromatin and thereby promotes H3K27 methylation. Nat. Struct. Mol. Biol. 24, 1039-1047 (2017)

15. Kim, J. M., Hong, K., Lee, J. H., Lee, S. \& Chang, N. Effect of folate deficiency on placental DNA methylation in hyperhomocysteinemic rats. J. Nutr. Biochem. 20, 172-176 (2009).

16. Zhang, D. et al. Hyperhomocysteinemia results from and promotes hepatocellular carcinoma via CYP450 metabolism by CYP2J2 DNA methylation. Oncotarget 8, 15377-15392 (2017).
17. Yang, A. N. et al. High-methionine diets accelerate atherosclerosis by HHcymediated FABP4 gene demethylation pathway via DNMT1 in ApoE(-/-) mice. FEBS Lett. 589, 3998-4009 (2015).

18. Li, F. et al. Histone Deacetylase 1 (HDAC1) Negatively Regulates Thermogenic Program in Brown Adipocytes via Coordinated Regulation of Histone $\mathrm{H} 3$ Lysine 27 (H3K27) Deacetylation and Methylation. J. Biol. Chem. 291, 4523-4536 (2016)

19. Girard, N. et al. 3-Deazaneplanocin A (DZNep), an inhibitor of the histone methyltransferase EZH2, induces apoptosis and reduces cell migration in chondrosarcoma cells. PLoS. One. 9, e98176 (2014).

20. Fillmore, C. M. et al. EZH2 inhibition sensitizes BRG1 and EGFR mutant lung tumours to Topoll inhibitors. Nature 520, 239-242 (2015).

21. Bayliss, J. et al. Lowered H3K27me3 and DNA hypomethylation define poorly prognostic pediatric posterior fossa ependymomas. Sci. Transl. Med. 8, 366ra161 (2016).

22. Zhou, Z., Garrow, T. A., Dong, X., Luchini, D. N. \& Loor, J. J. Hepatic Activity and Transcription of Betaine-Homocysteine Methyltransferase, Methionine Synthase, and Cystathionine Synthase in Periparturient Dairy Cows Are Altered to Different Extents by Supply of Methionine and Choline. J. Nutr. 147, 11-19 (2017).

23. Puri, P. \& Chandra, A. Autophagy modulation as a potential therapeutic target for liver diseases. J. Clin. Exp. Hepatol. 4, 51-59 (2014).

24. Zhao $Y$. et al. Homocysteine aggravates cortical neural cell injury through neuronal autophagy overactivation following rat cerebral ischemiareperfusion. International journal of molecular sciences 17, 1196 (2016).

25. Tazi, M. F. et al. Elevated Mirc1/Mir17-92 cluster expression negatively regulates autophagy and CFTR (cystic fibrosis transmembrane conductance regulator) function in CF macrophages. Autophagy 12, 2026-2037 (2016).

26. Xia, D. et al. MARCH2 regulates autophagy by promoting CFTR ubiquitination and degradation and PIK3CA-AKT-MTOR signaling. Autophagy 12, 1614-1630 (2016).

27. Luciani, A. et al. Defective CFTR induces aggresome formation and lung inflammation in cystic fibrosis through ROS-mediated autophagy inhibition Nat. Cell. Biol. 12, 863-875 (2010).

28. Tosco A. et al. A novel treatment of cystic fibrosis acting on-target: cysteamine plus epigallocatechin gallate for the autophagy-dependent rescue of class IImutated CFTR. Cell. Death. Differ. 23, 1380-1393 (2016).

29. Zhang, D. et al. Hyperhomocysteinemia results from and promotes hepatocellular carcinoma via CYP450 metabolism by CYP2J2 DNA methylation. Oncotarget 8, 15377-15392 (2017).

30. Lin, N. et al. Homocysteine induces cytotoxicity and proliferation inhibition in neural stem cells via DNA methylation in vitro. Febs. J. 281, 2088-2096 (2014).

31. Fu, Y., Wang, X. \& Kong, W. Hyperhomocysteinaemia and vascular injury: advances in mechanisms and drug targets. Br. J. Pharmacol. https://doi.org/ 10.1111/bph.13988 (2017).

32. Li, J. J. et al. Homocysteine Triggers Inflammatory Responses in Macrophages through Inhibiting CSE-H2S Signaling via DNA Hypermethylation of CSE Promoter. Int. J. Mol. Sci. 16, 12560-12577 (2015).

33. $\mathrm{Ma}$, S. et al. Hyperhomocysteinemia induces cardiac injury by up-regulation of p53-dependent Noxa and Bax expression through the p53 DNA methylation in ApoE(-/-) mice. Acta Biochim. Biophys. Sin. (Shanghai). 45, 391-400 (2013).

34. Pang, W. et al. The mPlrp2 and mClps genes are involved in the hydrolysis of retinyl esters in the mouse liver. J. Lipid Res. 52, 934-941 (2011).

35. Krishna, S. M., Dear, A., Craig, J. M., Norman, P. E. \& Golledge, J. The potentia role of homocysteine mediated DNA methylation and associated epigenetic changes in abdominal aortic aneurysm formation. Atherosclerosis $\mathbf{2 2 8}$ 295-305 (2013).

36. Tyagi, N. et al. Tetrahydrocurcumin ameliorates homocysteinylated cytochrome-c mediated autophagy in hyperhomocysteinemia mice after cerebral ischemia. J. Mol. Neurosci. 47, 128-138 (2012).

37. Whitington, P. F., Pan, X., Kelly, S., Melin-Aldana, H. \& Malladi, P. Gestational alloimmune liver disease in cases of fetal death. J. Pediatr. 159, 612-616 (2011).

38. Takamura, A. et al. Autophagy-deficient mice develop multiple liver tumors. Genes Dev. 25, 795-800 (2011).

39. Yang, A. et al. Modulation of FABP4 hypomethylation by DNMT1 and its inverse interaction with miR-148a/152 in the placenta of preeclamptic rats and HTR-8 cells. Placenta 46, 49-62 (2016).

40. Mosmann, T. Rapid colorimetric assay for cellular growth and survival: application to proliferation and cytotoxicity assays. J. Immunol. Methods 65, 55-63 (1983). 